\title{
THE BEHAVIOUR OF WEAK SOLUTIONS OF BOUNDARY VALUE PROBLEMS FOR LINEAR ELLIPTIC SECOND ORDER EQUATIONS IN UNBOUNDED CONE-LIKE DOMAINS
}

\author{
DAMIAN WIŚNIEWSKI
}

\begin{abstract}
We investigate the behaviour of weak solutions of boundary value problems (Dirichlet, Neumann, Robin and mixed) for linear elliptic divergence second order equations in domains extending to infinity along a cone. We find an exponent of the solution decreasing rate: we derive the estimate of the weak solution modulus for our problems near the infinity under assumption that leading coefficients of the equations do not satisfy the Dini-continuity condition.
\end{abstract}

\section{Introduction}

Many problems of mathematical physics lead one to consider the solution of boundary value problems for elliptic second order equations in unbounded domains and to study the behaviour of the solution at infinity, for instance stationary states, travelling waves, homogenization, boundary layer problems, Saint-Venant's principle and so on. Boundary value problems for linear elliptic equations in unbounded domains have applications to mechanics of inhomogeneous media [4]. Some problems in unbounded domains found applications in models in astrophysics (Eddington's model), are used in the analytic theory of polytropic ball model of stellar structures (Lane-Ritter-Emden theory)

Received: 24.02.2016. Revised: 25.05.2016. Accepted: 27.05.2016.

(2010) Mathematics Subject Classification: 35J25.

Key words and phrases: boundary value problems, weak solutions, second order elliptic linear equations, unbounded domains, Dini-continuity. 
and have numerous applications in natural sciences, e.g. in scalar field theory, in phase transition theory, in combustion theory, population dynamics [5], pseudo-plastic fluids [2, 3, 6], and ecology models [7].

Let $B_{1}(\mathcal{O})$ be the unit ball in $\mathbb{R}^{n}, n \geq 2$ with center at the origin $\mathcal{O}$ and $G \subset \mathbb{R}^{n} \backslash B_{1}(\mathcal{O})$ be an unbounded domain with the smooth boundary $\partial G$. We assume that there exists $R \gg 1$ such that $G=G_{0} \cup G_{R}$, where $G_{0}$ is a bounded domain in $\mathbb{R}^{n}, G_{R}=\left\{x=(r, \omega) \in \mathbb{R}^{n} \mid r \in(R, \infty)\right.$, $\omega \in \Omega \subset S^{n-1}, S^{n-1}$ is the unit sphere $\}$.

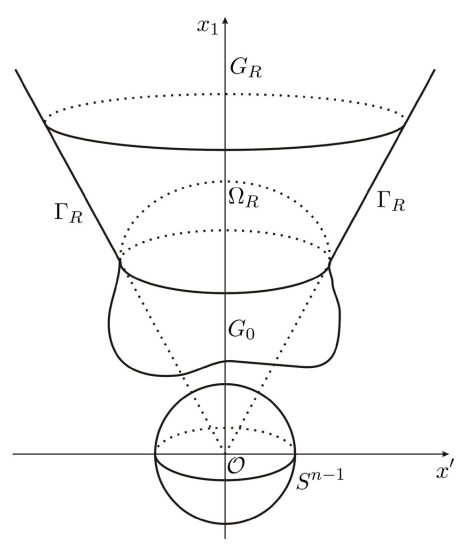

Figure 1. An unbounded cone-like domain

We consider the following linear problem

(L)

$$
\begin{cases}\frac{\partial}{\partial x_{i}}\left(a^{i j}(x) u_{x_{j}}\right)+b^{i}(x) u_{x_{i}}+c(x) u=f(x), & x \in G, \\ \alpha(x) \frac{\partial u}{\partial \nu}+\frac{1}{|x|} \gamma\left(\frac{x}{|x|}\right) u=g(x), & x \in \partial G, \\ \lim _{|x| \rightarrow \infty} u(x)=0, & \end{cases}
$$

here $\frac{\partial}{\partial \nu}=a^{i j}(x) \cos \left(\vec{n}, x_{i}\right) \frac{\partial}{\partial x_{j}}$, where $\vec{n}$ denotes the unit outward with respect to $G$ normal to $\partial G, \gamma(\omega)$ is positive bounded piecewise smooth function on $\partial \Omega$ such that $\gamma(\omega) \geq \gamma_{0}>0$ and

$$
\alpha(x)= \begin{cases}0, & \text { if } x \in \mathcal{D}, \\ 1, & \text { if } x \notin \mathcal{D},\end{cases}
$$

$\mathcal{D} \subseteq \partial G$ is the part of the boundary $\partial G$, where the Dirichlet boundary condition is posed. Thus, if $\mathcal{D}=\partial G$ then we have the Dirichlet problem, if $\mathcal{D}=\emptyset$ then $\alpha(x)=1$ and we have the Robin problem, if $\mathcal{D} \subset \partial G$ then we have the mixed problem. 
We introduce the following notations:

- $(r, \omega), \omega=\left(\omega_{1}, \omega_{2}, \ldots, \omega_{n-1}\right):$ spherical coordinates of $x \in \mathbb{R}^{n}$

$$
\begin{aligned}
x_{1} & =r \cos \omega_{1}, \\
x_{2} & =r \cos \omega_{2} \sin \omega_{1}, \\
\vdots & \\
x_{n-1} & =r \cos \omega_{n-1} \sin \omega_{n-2} \ldots \sin \omega_{1}, \\
x_{n} & =r \sin \omega_{n-1} \sin \omega_{n-2} \ldots \sin \omega_{1},
\end{aligned}
$$

where $r=|x|$;

$-\mathcal{C}$ : a rotational cone $\left\{x_{1}>r \cos \frac{\omega_{0}}{2}\right\}$

- $\partial \mathcal{C}$ : the lateral surface of $\mathcal{C}:\left\{x_{1}=r \cos \frac{\omega_{0}}{2}\right\}$;

$-\Omega$ : a domain on the unit sphere $S^{n-1}$ with smooth boundary $\partial \Omega$ obtained by the intersection of the cone $\mathcal{C}$ with the sphere $S^{n-1}$;

$-\partial \Omega=\partial \mathcal{C} \cap S^{n-1}$

- $G_{a}^{b}=\{(r, \omega) \mid a<r<b ; \omega \in \Omega\} \cap G$ : the layer in $\mathbb{R}^{n}$;

- $\Gamma_{a}^{b}=\{(r, \omega) \mid a<r<b ; \omega \in \partial \Omega\} \cap \partial G$ : the lateral surface of layer $G_{a}^{b}$;

$-G_{\varrho}=G_{\varrho}^{\infty} ; \quad \Gamma_{\varrho}=\Gamma_{\varrho}^{\infty}$.

We use standard function spaces: $C^{k}(\bar{G})$ with the norm $|u|_{k, \bar{G}}$, the Lebesgue space $L_{p}(G), p \geq 1$, with the norm $\|u\|_{p, G}$, the Sobolev space $W^{k, p}(G)$ with the norm $\|u\|_{p, k ; G}$. We define the weighted Sobolev spaces $V_{p, \alpha}^{k}(G)$ for an integer $k \geq 0$ and a real $\alpha$ as the closure of $C_{0}^{\infty}(\bar{G})$ with the finite norm

$$
\|u\|_{V_{p, \alpha}^{k}(G)}=\left(\int_{G} \sum_{|\beta|=0}^{k} r^{\alpha+p(|\beta|-k)}\left|D^{\beta} u\right|^{p} d x\right)^{\frac{1}{p}} .
$$

Throughout the paper we consider weak solutions of problem $\mathrm{L}$ defined as follows.

Definition 1.1. A function $u(x)$ is said to be a weak solution of problem (L) provided that $u(x) \in C^{0}(\bar{G}) \cap W^{1,2}(G), \lim _{|x| \rightarrow \infty} u(x)=0$ and satisfies the integral identity

$$
\begin{aligned}
\int_{G}\left\{a^{i j}(x) u_{x_{j}} \eta_{x_{i}}-b^{i}(x)\right. & \left.u_{x_{i}} \eta(x)-c(x) u \eta(x)\right\} d x+\int_{G} f(x) \eta(x) d x \\
& +\int_{\partial G} \alpha(x)\left\{\frac{1}{r} \gamma(\omega) u(x)-g(x)\right\} \eta(x) d s=0
\end{aligned}
$$

for all functions $\eta(x) \in C^{0}(\bar{G}) \cap W^{1,2}(G)$ such that $\lim _{|x| \rightarrow \infty} \eta(x)=0$. 
We assume that the following conditions are fulfilled:

(a) the condition of uniform ellipticity

$$
\begin{gathered}
\nu \xi^{2} \leq a^{i j}(x) \xi_{i} \xi_{j} \leq \mu \xi^{2} \quad \forall x \in \bar{G}, \quad \forall \xi \in \mathbb{R}^{n}, \\
\nu, \mu=\mathrm{const}>0 \quad \text { and } \quad \lim _{|x| \rightarrow \infty} a^{i j}(x)=\delta_{i}^{j},
\end{gathered}
$$

where $\delta_{i}^{j}$ is the Kronecker symbol;

(b) $a^{i j}(x) \in C^{0}(\bar{G}), b^{i}(x) \in L_{p}(G), p>n$; for them inequalities

$$
\sqrt{\sum_{i, j=1}^{n}\left|a^{i j}(x)-\delta_{i}^{j}\right|^{2}} \leq \mathcal{A}\left(\frac{1}{|x|}\right), \quad|x|\left(\sum_{i=1}^{n}\left|b^{i}(x)\right|^{2}\right)^{\frac{1}{2}} \leq \mathcal{A}\left(\frac{1}{|x|}\right)
$$

hold for $x \in G_{R}$, where $\mathcal{A}(t), t \geq 0$, is a monotonically increasing, nonnegative function, continuous at zero and $\lim _{r \rightarrow \infty} \mathcal{A}\left(\frac{1}{r}\right)=0$;

(c) $0 \geq c(x) \in L_{p / 2}(G) \cap L_{2}(G)$;

(d) $f(x) \in L_{p / 2}(G) \cap L_{2}(G)$;

(e) there exist numbers $f_{1} \geq 0, g_{1} \geq 0, s>0$ such that

$$
|f(x)| \leq f_{1}|x|^{-s-2}, \quad|g(x)| \leq g_{1}|x|^{-s-1} .
$$

In [8] we investigated the behaviour of weak solutions to the problem $(\mathrm{L})$ in a neighborhood of infinity assuming that the function $\mathcal{A}(t)$ is Dini-continuous at zero in the meaning of the following definition.

Definition 1.2. A function $\mathcal{A}(t)$ is called Dini continuous at zero if the integral

$$
\int_{0}^{d} \frac{\mathcal{A}(t)}{t} d t
$$

is finite for some $d>0$.

Our aim of this article is to derive the estimate of the weak solution modulus for our problem (L) near the infinity under assumption that leading coefficients of the equations do not satisfy the Dini-continuity condition. 


\section{Preliminaries}

Let $\vec{\nu}$ be the exterior normal to $\partial \mathcal{C}$ at points of $\partial \Omega$. We consider the eigenvalue problem for the Laplace-Beltrami operator on $\Delta_{\omega}$ the unit sphere

$(\mathrm{EVP})$

$$
\begin{cases}\triangle_{\omega} \psi+\vartheta \psi=0, & \omega \in \Omega, \\ \alpha(\omega) \frac{\partial \psi}{\partial \vec{\nu}}+\gamma(\omega) \psi(\omega)=0, & \omega \in \partial \Omega,\end{cases}
$$

which consists of the determination of all values $\vartheta$ (eigenvalues) for which EVP has weak solutions $\psi(\omega) \neq 0$ (eigenfunctions); here

$$
\alpha(\omega)= \begin{cases}0, & \text { if } \omega \in \partial_{\mathcal{D}} \Omega, \\ 1, & \text { if } \omega \in \partial \Omega \backslash \partial_{\mathcal{D}} \Omega,\end{cases}
$$

where $\partial_{\mathcal{D}} \Omega \subseteq \partial \Omega$ is the part of the boundary $\partial \Omega$ for which we consider the Dirichlet boundary condition.

Now we formulate three theorems which are essentially used in further consideration. All of them are proved in [8]. The first is the Hardy-FriedrichsWirtinger type inequality that is adapted to our problem (L).

Theorem 2.1. Let $u \in C^{0}\left(\overline{G_{R}}\right) \cap V_{2, \beta-2}^{1}\left(G_{R}\right)$ and $\gamma(\omega) \in C^{0}(\partial \Omega), \omega \in \partial \Omega$ be a positive bounded piecewise smooth function. Then

$$
\begin{aligned}
\int_{G_{R}} r^{\beta-4} u^{2} d x \leq & H\left(\lambda_{-}, n, \beta\right) \\
& \times\left\{\int_{G_{R}} r^{\beta-2}|\nabla u|^{2} d x+\int_{\Gamma_{R}} r^{\beta-3} \alpha(x) \gamma(\omega) u^{2}(x) d s\right\}, \\
H\left(\lambda_{-}, n, \beta\right)= & {\left[\left(\frac{\beta+n-4}{2}\right)^{2}+\lambda_{-}\left(\lambda_{-}+n-2\right)\right]^{-1}, \quad \beta \geq 4-n, } \\
\lambda_{-}= & \frac{2-n-\sqrt{(n-2)^{2}+4 \vartheta}}{2},
\end{aligned}
$$

where $\vartheta$ is the smallest positive eigenvalue of the eigenvalue problem (EVP).

REMARK 2.2. The proof of the existence of the smallest positive eigenvalue $\vartheta$ and associated eigenfunction $\psi(\omega)$ can be found in [1, §2.1]. 
The second is a theorem about the local boundedness at the infinity of weak solutions of problem (L).

THEOREM 2.3. Let $u(x)$ be a weak solution of problem $(\overline{\mathrm{L}})$, assumptions (a) -(d) be satisfied and $g(x) \in L_{\infty}(\partial G)$. Then the inequality

$\sup _{x \in G_{\varrho \varkappa}^{2 \varrho}}|u(x)| \leq \frac{\widetilde{C}}{(\varkappa-1)^{\tilde{n} / t}}\left\{\varrho^{-n / t}\|u\|_{t, G_{\varrho}^{2 \varrho}}+\varrho^{2(1-n / p)}\|f\|_{p / 2, G_{\varrho}^{2 \varrho}}+\varrho\|g\|_{\infty, \Gamma_{\varrho}^{2 \varrho}}\right\}$

holds for any $t>0, p>\widetilde{n}\left\{\begin{array}{ll}\geq n & \text { for } n \geq 3 \\ >2 & \text { for } n=2\end{array}, \quad \varkappa \in(1,2)\right.$ and $\varrho>R$.

The last theorem concerns the Cauchy problem for differential inequality.

THEOREM 2.4. Suppose that $U(\varrho)$ is a monotonically decreasing, nonnegative differentiable function defined on $[R, \infty), R \gg 1$, satisfying

$$
\left\{\begin{array}{l}
U^{\prime}(\varrho)+P(\varrho) U(\varrho)-Q(\varrho) \leq 0, \quad \varrho>R \\
U(R) \leq U_{0}
\end{array}\right.
$$

where $P(\varrho), Q(\varrho)$ are nonnegative continuous functions defined on $[R, \infty)$ and $U_{0}$ is a constant. Then

$$
U(\varrho) \leq U_{0} \exp \left(-\int_{R}^{\varrho} P(\sigma) d \sigma\right)+\int_{R}^{\varrho} Q(t) \exp \left(-\int_{t}^{\varrho} P(\sigma) d \sigma\right) d t .
$$

\section{Main results}

TheOrem 3.1. Let $u(x)$ be a weak solution of problem (L), $\lambda_{-}$be as in (2.2) and assumptions (a) -(e) be satisfied with $\mathcal{A}(t)$, which is a continuous at zero function, but not Dini continuous at zero. Then for all $\varepsilon>0$ there are $R \gg 1$ and a constant $C_{\varepsilon}$ depending only on $n, s, \lambda_{-}, \omega_{0}, R$ such that for all $\varrho>R$

$$
\begin{aligned}
& \int_{G_{\varrho}}\left(r^{2-n}|\nabla u|^{2}+r^{-n} u^{2}\right) d x+\int_{\Gamma_{\varrho}} r^{1-n} \gamma(\omega) u^{2}(x) \alpha(x) d s \\
& \quad \leq C_{\varepsilon}\left(\|u\|_{2, G}^{2}+f_{1}^{2}+\frac{1}{\gamma_{0}} g_{1}^{2}\right) \begin{cases}\varrho^{2 \lambda_{-}+\varepsilon} & \text { for } s \geq-\lambda_{-}, \\
\varrho^{-2 s} & \text { for } 0<s<-\lambda_{-} .\end{cases}
\end{aligned}
$$


Proof. As in the proof of Theorem 4.3 in [8] we obtain the differential inequality $\mathrm{CP}$ with

$$
\begin{aligned}
P(\varrho) & =-\frac{\frac{2 \lambda_{-}}{\varrho} \cdot\left[1-c_{1}\left(n, \lambda_{-}\right)\left(\delta+\mathcal{A}\left(\frac{1}{\varrho}\right)\right)\right]}{1+c_{2}\left(\lambda_{-}\right) \mathcal{A}\left(\frac{1}{\varrho}\right)}, \quad \delta>0, \\
Q(\varrho) & =-\frac{\frac{\lambda_{-}}{s} c_{0}\left(f_{1}^{2}+\frac{1}{\gamma_{0}} g_{1}^{2}\right) \cdot \delta^{-1} \varrho^{-2 s-1}}{1+c_{2}\left(\lambda_{-}\right) \mathcal{A}\left(\frac{1}{\varrho}\right)}, \quad \delta>0, \\
U_{0} & =\text { const } \cdot\left\{\int_{G}\left(u^{2}+r^{4-n} f^{2}\right) d x+\frac{1}{\gamma_{0}} \int_{\partial G} r^{3-n} g^{2} \alpha(x) d s\right\}, \\
U(\varrho) & =\int_{G_{\varrho}} r^{2-n}|\nabla u|^{2} d x+\int_{\Gamma_{\varrho}} r^{1-n} \gamma(\omega) u^{2}(x) \alpha(x) d s,
\end{aligned}
$$

where $c_{0}, c_{1}, c_{2}$ are positive constants. Since

$$
-P(\sigma)=\frac{2 \lambda_{-}}{\sigma} \cdot\left[1-\frac{c_{3} \mathcal{A}\left(\frac{1}{\sigma}\right)+\delta}{1+c_{2} \mathcal{A}\left(\frac{1}{\sigma}\right)}\right] \leq \frac{2 \lambda_{-}}{\sigma}\left(1-c_{3} \mathcal{A}\left(\frac{1}{\sigma}\right)-\delta\right)
$$

for all $\delta>0$, we have

$$
\begin{aligned}
-\int_{R}^{\varrho} P(\sigma) d \sigma & \leq 2 \lambda_{-} \int_{R}^{\varrho} \frac{1-c_{3} \mathcal{A}\left(\frac{1}{\sigma}\right)-\delta}{\sigma} d \sigma \\
& =2 \lambda_{-}(1-\delta) \ln \frac{\varrho}{R}-2 \lambda_{-} c_{3} \int_{R}^{\varrho} \frac{\mathcal{A}\left(\frac{1}{\sigma}\right)}{\sigma} d \sigma
\end{aligned}
$$

The mean value theorem for integrals yields

$$
\int_{R}^{\varrho} \frac{\mathcal{A}\left(\frac{1}{\sigma}\right)}{\sigma} d \sigma \leq \mathcal{A}\left(\frac{1}{R}\right) \ln \frac{\varrho}{R}
$$

Choosing $R \gg 1$ such that $2 c_{3} \mathcal{A}\left(\frac{1}{R}\right)<\delta$, we obtain

$$
\exp \left(-\int_{R}^{\varrho} P(\sigma) d \sigma\right) \leq\left(\frac{\varrho}{R}\right)^{2 \lambda_{-}\left(1-\frac{3}{2} \delta\right)}, \quad \delta>0 .
$$


Along similar lines

$$
\exp \left(-\int_{t}^{\varrho} P(\sigma) d \sigma\right) \leq\left(\frac{\varrho}{t}\right)^{2 \lambda_{-}\left(1-\frac{3}{2} \delta\right)}, \quad \delta>0
$$

Now we can estimate

$$
\begin{aligned}
\int_{R}^{\varrho} Q(t) \exp & \left(-\int_{t}^{\varrho} P(\sigma) d \sigma\right) d t \\
& \leq \frac{-\lambda_{-} c_{0}}{s \delta}\left(f_{1}^{2}+\frac{1}{\gamma_{0}} g_{1}^{2}\right) \int_{R}^{\varrho} t^{-2 s-1}\left(\frac{\varrho}{t}\right)^{2 \lambda_{-}\left(1-\frac{3}{2} \delta\right)} d t \\
& =c_{4} \varrho^{2 \lambda_{-}\left(1-\frac{3}{2} \delta\right)} \frac{\varrho^{-2 s-2 \lambda_{-}\left(1-\frac{3}{2} \delta\right)}-R^{-2 s-2 \lambda_{-}}\left(1-\frac{3}{2} \delta\right)}{-2 s-2 \lambda_{-}\left(1-\frac{3}{2} \delta\right)} \\
& \leq c_{5} \begin{cases}\varrho^{-2 s} & \text { for } s<-\lambda_{-}, \delta \neq \frac{2}{3} \frac{s+\lambda_{-}}{\lambda_{-}}, \\
\varrho^{2 \lambda_{-}\left(1-\frac{3}{2} \delta\right)} & \text { for } s \geq-\lambda_{-} .\end{cases}
\end{aligned}
$$

Next, from 2.3 we deduce

$$
\begin{aligned}
U(\varrho) & \leq U_{0}\left(\frac{\varrho}{R}\right)^{2 \lambda_{-}\left(1-\frac{3}{2} \delta\right)}+c_{5} \begin{cases}\varrho^{-2 s} & \text { for } s<-\lambda_{-}, \\
\varrho^{2 \lambda_{-}\left(1-\frac{3}{2} \delta\right)} & \text { for } s \geq-\lambda_{-},\end{cases} \\
& \leq C_{\varepsilon}\left\{\begin{array}{ll}
\varrho^{-2 s} & \text { for } s<-\lambda_{-}, \\
\varrho^{2 \lambda_{-}+\varepsilon} & \text { for } s \geq-\lambda_{-},
\end{array} \text {for all } \varepsilon>0 .\right.
\end{aligned}
$$

Thus, in virtue of 2.1 for $\beta=4-n$, Theorem 3.1 is proved.

We can now correct Theorem 3.1 in the case $s=-\lambda_{-}$, if $\mathcal{A}\left(\frac{1}{r}\right) \sim \frac{1}{\ln r}$.

TheOREM 3.2. Let $u(x)$ be a weak solution of problem (L), $\lambda_{-}$be as in (2.2) and assumptions (a)-(e) be satisfied with $\mathcal{A}\left(\frac{1}{r}\right) \sim \frac{1}{\ln r}$. Then there exist $R \gg 1$ and constants $C>0, c>0$ depending only on $n, \lambda_{-}, \omega_{0}, R$ such that for all $\varrho>R$

$$
\begin{aligned}
\int_{G_{\varrho}}\left(r^{2-n}|\nabla u|^{2}+r^{-n} u^{2}\right) d x+ & \int_{\Gamma_{\varrho}} r^{1-n} \gamma(\omega) u^{2}(x) \alpha(x) d s \\
& \leq C\left(\|u\|_{2, G}^{2}+f_{1}^{2}+\frac{1}{\gamma_{0}} g_{1}^{2}\right) \varrho^{2 \lambda_{-}} \ln ^{2+c} \varrho .
\end{aligned}
$$


Proof. In the case $s=-\lambda_{-}$we can estimate

$$
\begin{aligned}
-P(\varrho) & \leq \frac{2 \lambda_{-}(1-\delta)}{\varrho}-\frac{2 \lambda_{-} c_{3} \mathcal{A}\left(\frac{1}{\varrho}\right)}{\varrho}, \\
Q(\varrho) & \leq c_{6}\left(f_{1}^{2}+\frac{1}{\gamma_{0}} g_{1}^{2}\right) \varrho^{2 \lambda_{-}-1} \delta^{-1} .
\end{aligned}
$$

Choosing now $\delta=\delta(\varrho)=-\frac{1}{2 \lambda_{-} \ln \varrho}$, we can conclude from assumption of theorem, that $\mathcal{A}\left(\frac{1}{\varrho}\right) \sim \delta(\varrho)$. We can estimate as follows

$$
\begin{aligned}
-\int_{R}^{\varrho} P(\sigma) d \sigma & \leq 2 \lambda_{-}\left(\int_{R}^{\varrho} \frac{1}{\sigma} d \sigma-\int_{R}^{\varrho} \frac{c_{3} \mathcal{A}\left(\frac{1}{\sigma}\right)+\delta(\sigma)}{\sigma} d \sigma\right) \\
& \leq \ln \left(\frac{\varrho}{R}\right)^{2 \lambda_{-}}-2 \lambda_{-} c_{7} \int_{R}^{\varrho} \frac{d \sigma}{\sigma \ln \sigma} \\
& =\ln \left(\frac{\varrho}{R}\right)^{2 \lambda_{-}}-2 \lambda_{-} c_{7} \ln (\ln \varrho),
\end{aligned}
$$

which implies that

$$
\exp \left(-\int_{R}^{\varrho} P(\sigma) d \sigma\right) \leq\left(\frac{\varrho}{R}\right)^{2 \lambda_{-}}(\ln \varrho)^{c} .
$$

In this way we have

$$
\exp \left(-\int_{t}^{\varrho} P(\sigma) d \sigma\right) \leq\left(\frac{\varrho}{t}\right)^{2 \lambda_{-}}(\ln \varrho)^{c} .
$$

We also have

$$
\begin{aligned}
\int_{R}^{\varrho} Q(t) \exp \left(-\int_{t}^{\varrho} P(\sigma) d \sigma\right) d t & \leq c_{8} \int_{R}^{\varrho} \delta^{-1} t^{2 \lambda_{-}-1}\left(\frac{\varrho}{t}\right)^{2 \lambda_{-}}(\ln \varrho)^{c} d t \\
& =-2 \lambda_{-} c_{8}(\ln \varrho)^{c} \varrho^{2 \lambda_{-}} \int_{R}^{\varrho} \frac{\ln t}{t} d t \\
& =-\left.\lambda_{-} c_{8}(\ln \varrho)^{c} \varrho^{2 \lambda_{-}}(\ln t)^{2}\right|_{t=R} ^{t=\varrho} \\
& \leq-\lambda_{-} c_{8}(\ln \varrho)^{c+2} \varrho^{2 \lambda_{-}}
\end{aligned}
$$


Now, using (2.3) yields

$$
U(\varrho) \leq U_{0}\left(\frac{\varrho}{R}\right)^{2 \lambda_{-}}(\ln \varrho)^{c}-\lambda_{-} c_{8} \varrho^{2 \lambda_{-}}(\ln \varrho)^{c+2} \leq C \varrho^{2 \lambda_{-}}(\ln \varrho)^{c+2} .
$$

Regarding to 2.1 for $\beta=4-n$, we obtain the statement of our theorem.

Now we are able to prove the following theorems.

THEOREM 3.3. Let $u(x)$ be a weak solution of problem (L), $\lambda_{-}$be as in (2.2) and assumptions (a)-(e) be satisfied with $\mathcal{A}(t)$, which is a continuous at zero function, but not Dini continuous at zero. Then for all $\varepsilon>0$ there are $R \gg 1$ and a constant $C_{\varepsilon}>0$ such that for all $x \in G_{R}$

$$
|u(x)| \leq C_{\varepsilon}\left(\|u\|_{2, G}+f_{1}+\frac{1}{\sqrt{\gamma_{0}}} g_{1}\right) \begin{cases}|x|^{\lambda_{-}+\varepsilon}, & \text { if } s \geq-\lambda_{-}, \\ |x|^{-s}, & \text { if } 0<s<-\lambda_{-} .\end{cases}
$$

Proof. We define the function

$$
\psi(\varrho)= \begin{cases}\varrho^{\lambda_{-}+\varepsilon}, & \text { if } s \geq-\lambda_{-} \\ \varrho^{-s}, & \text { if } 0<s<-\lambda_{-} .\end{cases}
$$

By Theorem 2.3 devoted to the local bound of the weak solution modulus, we have

$$
\sup _{G_{\frac{3}{2} \varrho}^{2 \varrho}}|u(x)| \leq \widetilde{C}\left\{\varrho^{-n / 2}\|u\|_{2, G_{\varrho}^{2 \varrho}}+\varrho^{2(1-n / p)}\|f\|_{p / 2, G_{\varrho}^{2 \varrho}}+\varrho\|g\|_{\infty, \Gamma_{\varrho}^{2 \varrho}}\right\}
$$

where $2 n \geq p>n \geq 2$. Now by Theorem 3.1 we have

$$
\begin{aligned}
\varrho^{-n / 2}\|u\|_{2, G_{\varrho}^{2} \varrho} & \leq 2^{n / 2}\left(\int_{G_{\varrho}^{2 \varrho}} r^{-n} u^{2}(x) d x\right)^{1 / 2} \\
& \leq C_{\varepsilon}\left(\|u\|_{2, G}+f_{1}+\frac{1}{\sqrt{\gamma_{0}}} g_{1}\right) \psi(\varrho) .
\end{aligned}
$$

Further, by assumptions (e) we obtain

$$
\varrho^{2(1-n / p)}\|f\|_{p / 2, G_{\varrho}^{2 \varrho}}+\varrho\|g\|_{\infty, \Gamma_{\varrho}^{2 \varrho}} \leq c_{9}\left(f_{1}+\frac{1}{\sqrt{\gamma_{0}}} g_{1}\right) \psi(\varrho) .
$$


It follows from $3.2-3.4$ that

$$
\sup _{x \in G_{3 / 2 \varrho}^{2 \varrho}}|u(x)| \leq C_{\varepsilon}\left\{\|u\|_{2, G}+f_{1}+\frac{1}{\gamma_{0}} g_{1}\right\} \psi(\varrho) .
$$

Putting now $|x|=\frac{7}{4} \varrho$ we finally obtain the desired estimate.

THEOREM 3.4. Let $u(x)$ be a weak solution of problem (L), $\lambda_{-}$be as in (2.2) and assumptions (a)-(e) be satisfied with $\mathcal{A}\left(\frac{1}{r}\right) \sim \frac{1}{\ln r}$. Then there exist $R \gg 1$ and constants $C_{0}>0, c>0$ depending only on $n, \lambda_{-}, f_{1}, g_{1}, \gamma_{0}, \nu, \mu, t, p$, $\left\|\sum_{i=1}^{n}\left|b^{i}(x)\right|^{2}\right\|_{L_{p / 2}(G)}$ such that for all $x \in G_{R}$

$$
|u(x)| \leq C_{0}\left(\|u\|_{2, G}+f_{1}+\frac{1}{\sqrt{\gamma_{0}}} g_{1}\right)|x|^{\lambda_{-}}(\ln |x|)^{c+1}
$$

Proof. One needs to repeat the proof of Theorem 3.3 taking

$$
\psi(\varrho)=\varrho^{\lambda_{-}}(\ln \varrho)^{c+1}
$$

and apply Theorem 3.2 .

REMARK 3.5. Now we can compare the obtained results 3.1 with those ones of [8]. More precisely, if the function $\mathcal{A}(t)$ from assumption (b) is Dinicontinuous at zero, then we get from [8] that

$$
|u(x)| \leq C_{0}\left(\|u\|_{2, G}+f_{1}+\frac{1}{\sqrt{\gamma_{0}}} g_{1}\right) \begin{cases}|x|^{\lambda_{-}}, & \text {if } s>-\lambda_{-} \\ |x|^{\lambda_{-}} \ln |x|, & \text { if } s=-\lambda_{-} \\ |x|^{-s}, & \text { if } 0<s<-\lambda_{-}\end{cases}
$$

First of all, the results coincide only when $0<s<-\lambda$. We can easily see that, near the infinity, for $s \geq-\lambda_{-}$(taking into account the fact that $\lim _{k \rightarrow+\infty} \frac{\ln k}{k^{\varepsilon}}=0$ for all $\varepsilon>0$ ) the weak solution modulus are estimated by bigger term in (3.1). 


\section{Example}

Suppose that $n=2$. Let

$$
G_{R}=\left\{(r, \omega) \mid r>R ;-\frac{\omega_{0}}{2}<\omega<\frac{\omega_{0}}{2} ; \quad \omega_{0} \in(0, \pi)\right\}
$$

and

$$
\Gamma_{R}^{ \pm}=\left\{(r, \omega) \mid r>R, \omega= \pm \frac{\omega_{0}}{2}\right\}
$$

We put

$$
\left.\gamma(\omega)\right|_{\omega= \pm \frac{\omega_{0}}{2}}=\gamma_{ \pm}=\mathrm{const} \geq 0 \quad \text { and }\left.\quad \alpha(x)\right|_{\Gamma_{R}^{ \pm}}=\alpha_{ \pm} \in\{0,1\} .
$$

We can easily observe that the function $u(r, \omega)=r^{\lambda_{-}} \psi(\omega), \lambda_{-}<0$ is a solution of the problem

$$
\left\{\begin{array}{l}
\Delta u=0, \quad x \in G_{R}, \\
\left.\left(\alpha_{ \pm} \frac{\partial u}{\partial n}+\frac{1}{r} \gamma_{ \pm} u\right)\right|_{\Gamma_{R}^{ \pm}}=0,
\end{array}\right.
$$

if $\lambda_{-}^{2}$ is the least positive eigenvalue of the problem

$$
\left\{\begin{array}{l}
\psi^{\prime \prime}+\lambda_{-}^{2} \psi=0, \quad \omega \in\left(-\frac{\omega_{0}}{2}, \frac{\omega_{0}}{2}\right) \\
\left.\left( \pm \alpha_{ \pm} \psi^{\prime}+\gamma_{ \pm} \psi\right)\right|_{\omega= \pm \frac{\omega_{0}}{2}}=0
\end{array}\right.
$$

and $\psi(\omega)$ is a regular eigenfunction associated with $\lambda_{-}^{2}$. Precisely $\lambda_{-}<0$ is defined via the transcendent equation

$$
\tan \left(\omega_{0} \lambda_{-}\right)=\frac{\lambda_{-}\left(\alpha_{+} \gamma_{-}+\alpha_{-} \gamma_{+}\right)}{\lambda_{-}^{2} \alpha_{+} \alpha_{-}-\gamma_{+} \gamma_{-}},
$$

while eigenfunction

$$
\psi(\omega)=\lambda_{-} \alpha_{+} \cos \left[\lambda_{-}\left(\omega-\frac{\omega_{0}}{2}\right)\right]-\gamma_{+} \sin \left[\lambda_{-}\left(\omega-\frac{\omega_{0}}{2}\right)\right] .
$$


EXAMPLE. We consider the following problem

$$
\left\{\begin{array}{l}
\frac{\partial}{\partial x_{i}}\left(a^{i j}(x) u_{x_{j}}\right)+b^{i}(x) u_{x_{i}}=0, \quad x \in G_{R} \\
\left.\left(\alpha_{ \pm} \frac{\partial u}{\partial n}+\frac{1}{r} \gamma_{ \pm} u\right)\right|_{\Gamma_{R}^{ \pm}}=0, \quad \gamma_{ \pm}>0
\end{array}\right.
$$

in the domain $G_{R}$, where

$$
\begin{gathered}
a^{11}(x)=1-\frac{2}{1+\lambda_{-}} \cdot \frac{x_{2}^{2}}{r^{2} \ln \frac{1}{r}}, \quad a^{22}(x)=1-\frac{2}{1+\lambda_{-}} \cdot \frac{x_{1}^{2}}{r^{2} \ln \frac{1}{r}}, \\
a^{12}(x)=a^{21}(x)=\frac{2}{1+\lambda_{-}} \cdot \frac{x_{1} x_{2}}{r^{2} \ln \frac{1}{r}}, \quad \lambda_{-}<-1, \\
\lim _{|x| \rightarrow \infty} a^{i j}(x)=\delta_{i}^{j}, \quad(i, j=1,2), \\
b^{1}=-\frac{1}{r} \mathcal{A}\left(\frac{1}{r}\right) \cos \omega, \quad b^{2}=-\frac{1}{r} \mathcal{A}\left(\frac{1}{r}\right) \sin \omega .
\end{gathered}
$$

Let us check that the function

$$
u(r, \omega)=r^{\lambda_{-}} \ln ^{\frac{\lambda_{-}-1}{\lambda_{-}+1}}\left(\frac{1}{r}\right) \cdot \psi(\omega)
$$

with $\lambda_{-}<-1$ and $\psi(\omega)$ defined by (4.1)- 4.2 is a solution of our problem. Firstly, we prove that $\frac{\partial}{\partial x_{i}}\left(a^{i j}(x) u_{x_{j}}\right)+b^{i}(x) u_{x_{i}}=0$. Indeed, differentiating we get $\frac{\partial}{\partial x_{i}}\left(a^{i j}(x) u_{x_{j}}\right)=\frac{\partial a^{i j}(x)}{\partial x_{i}} u_{x_{j}}+a^{i j}(x) u_{x_{j} x_{i}}$. Setting $F(r)=\frac{2}{\lambda_{-}+1} \frac{1}{\ln \frac{1}{r}}$ and calculating derivatives in the polar coordinates, in virtue of

$$
\frac{\partial}{\partial x_{1}}=\frac{\partial}{\partial r} \cos \omega-\frac{\partial}{\partial \omega} \frac{\sin \omega}{r}, \quad \frac{\partial}{\partial x_{2}}=\frac{\partial}{\partial r} \sin \omega+\frac{\partial}{\partial \omega} \frac{\cos \omega}{r},
$$

we have

$$
\begin{aligned}
& \frac{\partial a^{11}(x)}{\partial x_{1}}=-\frac{1}{2} F^{\prime}(r) \sin \omega \sin 2 \omega+\frac{1}{r} F(r) \sin \omega \sin 2 \omega \\
& \frac{\partial a^{12}(x)}{\partial x_{1}}=\frac{1}{2} F^{\prime}(r) \cos \omega \sin 2 \omega-\frac{1}{r} F(r) \sin \omega \cos 2 \omega \\
& \frac{\partial a^{21}(x)}{\partial x_{2}}=\frac{1}{2} F^{\prime}(r) \sin \omega \sin 2 \omega+\frac{1}{r} F(r) \cos \omega \cos 2 \omega
\end{aligned}
$$




$$
\frac{\partial a^{22}(x)}{\partial x_{2}}=-\frac{1}{2} F^{\prime}(r) \cos \omega \sin 2 \omega+\frac{1}{r} F(r) \cos \omega \sin 2 \omega
$$

Therefore, by

$$
\begin{aligned}
& \frac{\partial^{2}}{\partial x_{1}^{2}}=\cos ^{2} \omega \frac{\partial^{2}}{\partial r^{2}}-\frac{\sin 2 \omega}{r} \frac{\partial^{2}}{\partial r \partial \omega}+\frac{\sin ^{2} \omega}{r^{2}} \frac{\partial^{2}}{\partial \omega^{2}}+\frac{\sin ^{2} \omega}{r} \frac{\partial}{\partial r}+\frac{\sin 2 \omega}{r^{2}} \frac{\partial}{\partial \omega} \\
& \frac{\partial^{2}}{\partial x_{2}^{2}}=\sin ^{2} \omega \frac{\partial^{2}}{\partial r^{2}}+\frac{\sin 2 \omega}{r} \frac{\partial^{2}}{\partial r \partial \omega}+\frac{\cos ^{2} \omega}{r^{2}} \frac{\partial^{2}}{\partial \omega^{2}}+\frac{\cos ^{2} \omega}{r} \frac{\partial}{\partial r}-\frac{\sin 2 \omega}{r^{2}} \frac{\partial}{\partial \omega} \\
& \frac{\partial^{2}}{\partial x_{1} \partial x_{2}}= \frac{\partial^{2}}{\partial x_{2} \partial x_{1}}=\frac{1}{2} \sin 2 \omega \frac{\partial^{2}}{\partial r^{2}}+\frac{\cos 2 \omega}{r} \frac{\partial^{2}}{\partial r \partial \omega} \\
&-\frac{1}{2} \frac{\sin 2 \omega}{r^{2}} \frac{\partial^{2}}{\partial \omega^{2}}-\frac{1}{2} \frac{\sin 2 \omega}{r} \frac{\partial}{\partial r}+\frac{\cos 2 \omega}{r^{2}} \frac{\partial}{\partial \omega}
\end{aligned}
$$

and

$$
\begin{aligned}
\frac{\partial u}{\partial r} & =r^{\lambda_{-}-1}\left(\ln \frac{1}{r}\right)^{-\frac{2}{\lambda_{-}+1}}\left[\lambda_{-} \ln \frac{1}{r}-\frac{\lambda_{-}-1}{\lambda_{-}+1}\right] \psi(\omega), \\
\frac{\partial^{2} u}{\partial r^{2}} & =\left(\lambda_{-}-1\right) r^{\lambda_{-}-2}\left(\ln \frac{1}{r}\right)^{\frac{-\lambda_{-}-3}{\lambda_{-}+1}}\left[\lambda_{-} \ln ^{2} \frac{1}{r}-\frac{2 \lambda_{-}-1}{\lambda_{-}+1} \ln \frac{1}{r}-\frac{2}{\left(\lambda_{-}+1\right)^{2}}\right] \psi(\omega), \\
\frac{\partial u}{\partial \omega} & =r^{\lambda_{-}}\left(\ln \frac{1}{r}\right)^{\frac{\lambda_{-}-1}{\lambda_{-}+1}} \psi^{\prime}(\omega), \\
\frac{\partial^{2} u}{\partial \omega^{2}} & =r^{\lambda_{-}}\left(\ln \frac{1}{r}\right)^{\frac{\lambda_{-}-1}{\lambda_{-}+1}} \psi^{\prime \prime}(\omega),
\end{aligned}
$$

we obtain

$$
\begin{aligned}
& \sum_{i, j=1}^{2}\left(\frac{\partial a^{i j}(x)}{\partial x_{i}} u_{x_{j}}+a^{i j}(x) u_{x_{j} x_{i}}\right)+b^{i}(x) u_{x_{i}} \\
& =\frac{\partial^{2} u}{\partial r^{2}}+[1-F(r)] \frac{1}{r^{2}} \frac{\partial^{2} u}{\partial \omega^{2}}+\frac{\partial u}{\partial r}+c(x) u(x) \\
& =r^{\lambda_{-}-2}\left(\ln \frac{1}{r}\right)^{\frac{-2}{\lambda_{-}+1}}\left[\ln \frac{1}{r}-\frac{2}{\lambda_{-}+1}\right]\left(\psi^{\prime \prime}(\omega)+\lambda_{-}^{2} \psi(\omega)\right)=0 .
\end{aligned}
$$

Boundary conditions follow immediately from the formula $\left.\frac{\partial u}{\partial n}\right|_{\Gamma_{R}^{ \pm}}= \pm\left.\frac{1}{r} \frac{\partial u}{\partial \omega}\right|_{\Gamma_{R}^{ \pm}}$. 
For $R>\exp \left(\frac{4}{-\lambda_{-}-1}\right)$ the condition of uniform ellipticity is fulfilled with constants $\mu=1$ and $\nu=1+\frac{4}{\left(\lambda_{-}+1\right) \ln R}, a^{i j}(x)$ are continuous at the infinity, but $\mathcal{A}\left(\frac{1}{r}\right)=\frac{2}{\lambda_{-}+1} \ln ^{-1}\left(\frac{1}{r}\right) \sim \frac{1}{\ln r}$, i.e. the function $\mathcal{A}(r)$ does not satisfy the Dini condition at zero. We can easily see that the solution (4.3) satisfy $|u(r, \omega)| \leq C \cdot|x|^{\lambda_{-}}(\ln |x|)^{1-\frac{2}{\lambda_{-}+1}}$, which coincide with result of Theorem 3.4 .

\section{References}

[1] Borsuk M.V., Transmission problems for elliptic second-order equations in non-smooth domains, Birkhäuser, Basel, 2010.

[2] Hernández J., Mancebo F.J., Vega J.M., On the linearization of some singular, nonlinear elliptic problems and applications, Ann. Inst. H. Poincaré Anal. Non Linéaire 19 (2002), 777-813.

[3] Lazer A.C., McKenna P.J., On a singular nonlinear elliptic boundary-value problem, Proc. Amer. Math. Soc. 111 (1991), 721-730.

[4] Mityushev V., Adler P., Darcy flow around a two-dimensional lens, I. Phys. A: Math. Gen. 39 (2006), 3545-3560.

[5] Murray J.D., Mathematical Biology, Springer, Berlin, 1993.

[6] Nachman A., Callegari A., A nonlinear singular boundary value problem in the theory of pseudoplastic fluids, SIAM J. Appl. Math. 38 (1980), 275-281.

[7] Okubo A., Levin S.A., Diffusion and Ecological Problems: Modern Prospectives, Springer, New York, 2001.

[8] Wiśniewski D., Boundary value problems for a second-order elliptic equation in unbounded domains, Ann. Univ. Paed. Cracov. Studia Math. 9 (2010), 87-122.

Faculty of Mathematics and Computer Science

University of Warmia and MaZury in Olszty

SLONECZNA 54

10-710 OLSZTyn

POLAND

e-mail: dawi@matman.uwm.edu.pl 